\title{
Is TEVAR Really Needed for Uncomplicated Type B Aortic Dissection?
}

\author{
Sven Zhen Cian Patrick Tan ${ }^{1}$, Hazem Elsantawy², and Amr Abdelhaliem ${ }^{3}$ \\ ${ }^{1}$ Queen Mary University of London Barts and The London School of Medicine and \\ Dentistry \\ ${ }^{2}$ Royal Blackburn Hospital \\ ${ }^{3}$ East Lancashire Hospitals NHS Trust
}

July 8, 2021

\begin{abstract}
Thoracic endovascular aortic repair (TEVAR) has quickly become the mainstay of treatment for acute aortic dissection, in particular cases of acute complicated Stanford Type B dissection (co-TBAD). Necessarily, TEVAR carries with it the risk of postoperative complications, including stroke and renal failure. As a result, the management of patients with uncomplicated type B aortic dissection (un-TBAD), which is generally accepted as being less severe, are safely managed via optimal medical therapy (OMT) alone. However, despite OMT, patients with un-TBAD are at substantial risk of severe disease progression requiring delayed intervention. The cost-benefit ratio associated with TEVAR for un-TBAD is therefore of key interest. Howard and colleagues produced a fascinating systematic review and meta-analysis investigating the clinical outcomes of TEVAR for complicated and uncomplicated TBAD. Their data suggests that there is no significant difference in in-hospital mortality or 5-year survival between TEVAR for un-TBAD and co-TBAD, although the 30-day mortality rate appeared to be higher in the co-TBAD cohort. Patients with co-TBAD appeared to also be at a higher risk of postoperative stroke and TEVAR endoleak, while un-TBAD patients were at a higher risk of postoperative renal failure. Further prospective research into these relationships are recommended to fully elucidate the comparative efficacies of TEVAR for un-TBAD and co-TBAD.
\end{abstract}

\section{Is TEVAR Really Needed for Uncomplicated Type B Aortic Dissection?}

Running title: Endovascular Management of Type B Aortic Dissection

Sven Zhen Cian Patrick Tan ${ }^{1} \mid$ Hazem El Santawy ${ }^{2}$ Amr Abdelhaliem²

1: Barts and The London School of Medicine and Dentistry, Queen Mary University of London, E1 2AD, UK

2: Department of Vascular and Endovascular Surgery, Royal Blackburn Teaching Hospital, Haslingden Road, BB2 3HH, UK

\section{Correspondence:}

Amr Abdelhaliem

Department of Vascular and Endovascular Surgery, Royal Blackburn Teaching Hospital, Haslingden Road, BB2 3HH, UK

Email: amrhaliem@gmail.com

Funding declarations: None to be declared 
Conflicts of interest: None to be declared

Keywords: TBAD, Complicated, Uncomplicated, TEVAR, OMT, Endovascular

\begin{abstract}
Thoracic endovascular aortic repair (TEVAR) has quickly become the mainstay of treatment for acute aortic dissection, in particular cases of acute complicated Stanford Type B dissection (co-TBAD). Necessarily, TEVAR carries with it the risk of postoperative complications, including stroke and renal failure. As a result, the management of patients with uncomplicated type B aortic dissection (un-TBAD), which is generally accepted as being less severe, are safely managed via optimal medical therapy (OMT) alone. However, despite OMT, patients with un-TBAD are at substantial risk of severe disease progression requiring delayed intervention. The cost-benefit ratio associated with TEVAR for un-TBAD is therefore of key interest. Howard and colleagues produced a fascinating systematic review and meta-analysis investigating the clinical outcomes of TEVAR for complicated and uncomplicated TBAD. Their data suggests that there is no significant difference in in-hospital mortality or 5-year survival between TEVAR for un-TBAD and co-TBAD, although the 30-day mortality rate appeared to be higher in the co-TBAD cohort. Patients with co-TBAD appeared to also be at a higher risk of postoperative stroke and TEVAR endoleak, while un-TBAD patients were at a higher risk of postoperative renal failure. Further prospective research into these relationships are recommended to fully elucidate the comparative efficacies of TEVAR for un-TBAD and co-TBAD.
\end{abstract}

\title{
Main Text
}

We read with interest the manuscript by Howard and Sheridan titled "TEVAR for Complicated and Uncomplicated Type B Aortic Dissection - Systematic Review and Meta-Analysis." ${ }^{1}$ The authors explained with profound clarity the respective pathological processes underpinning both complicated and uncomplicated type $\mathrm{B}$ aortic dissection (co-TBAD and un-TBAD respectively), as well as the different clinical manifestations associated with either pathology. ${ }^{1}$ In addition, the authors outline the role played by thoracic endovascular aortic repair (TEVAR) in the surgical management of this complex condition. ${ }^{1}$ Howard et al. highlight that although both co-TBAD and un-TBAD represent complex structural pathologies of the thoracic aorta, un-TBAD can often be sufficiently managed with pharmacotherapy to control blood pressure. ${ }^{1}$ On the other hand, co-TBAD usually warrants endovascular or open surgical intervention to stabilise the aortic wall and prevent disease progression. ${ }^{1,2}$ Because the clinical outcomes of the surgical management of both un-TBAD and co-TBAD vary from centre to centre, the investigators undertook a systematic review and meta-analysis to evaluate the role of TEVAR for un-TBAD and co-TBAD, in terms of in-hospital and five-year mortality, and postoperative complications. ${ }^{1}$ Recent advancements in TEVAR have given rise to improved outcomes in patients suffering from both un-TBAD and co-TBAD. ${ }^{3}$ Whereas TEVAR is widely regarded as standard acute treatment for co-TBAD, this has led clinicians to question whether a more aggressive, endovascular approach may be more beneficial for patients with un-TBAD than the traditional, conservative, pharmacotherapeutic approach. ${ }^{1,4}$ The decision to investigate the role of TEVAR for un-TBAD and co-TBAD via a systematic review and meta-analysis is therefore prudent.

The study benefits from robust and appropriate inclusion and exclusion criteria, both of which are clearly outlined in the manuscript. By excluding studies focused on patients with type A aortic dissection, or patients treated with an open surgical or hybrid surgical-endovascular approach, Howard and colleagues maintained a respectable level of focus and specificity without compromising sample size (16,104 patients across 89 studies were included). ${ }^{1}$ Howard et al. also carried out appropriate statistical analyses: the t-test was used to ascertain the relationship between continuous variables while the chi square test was used for discreet, categorical variables. ${ }^{1}$ In doing so, the investigators suggest that there was a statistically significant difference in the incidence of post-TEVAR complications (a continuous variable) between co-TBAD and unTBAD, such as stroke $(5.85 \%$ vs $3.92 \%, \mathrm{P}<0.01)$ and renal failure $(7.23 \%$ vs $11.38 \%, \mathrm{P}<0.01) .{ }^{1}$ Similarly, the results suggest that co-TBAD was more associated with certain comorbidities (discreet variables) than un-TBAD, such as diabetes ( $8.77 \%$ vs $1.37 \%, \mathrm{P}<0.01)$ and Marfan syndrome $(3.47 \%$ vs $1.37 \%, \mathrm{P}<0.01) .{ }^{1}$ Certainly, revealing such relationships may well allow Howard and colleagues' study to guide physicians in 
clinical decision making.

The results derived from the study could further be strengthened by the inclusion of analyses that would evaluate the hetero- or homogeneity of the data, and the presence of publication bias amongst the studies included. ${ }^{5}$ Deriving a Cochran's $Q$ value could determine whether the data included is substantially heterogenous, and indeed deriving a Higgins $I^{2}$ value would help to determine the extent to which said variation results from true heterogeneity rather than chance. ${ }^{5} \mathrm{~A}$ meta-regression or subgroup analysis could then be carried out to explore the nature and clinical significance thereof.

In addition, it would also be worthwhile to ascertain the degree to which the publications included in metaanalysis were subject to publication bias. A subjective, visual evaluation thereof could be achieved via the inclusion of a funnel plot; alternatively, Egger's test could be performed to objectively elucidate this. ${ }^{6}$ For example, in their investigation of neurological complications following frozen elephant trunk repair for aortic dissection, Mousavizadehet al. were able to determine via Higgins $I^{2}$ that most of their data, bar a select few outcomes, were subject to significant heterogeneity; a funnel plot and Egger's test also revealed a substantial level of publication bias in each outcome. ${ }^{7}$ This made clearer the limitations associated with Mousavizadeh and colleagues' work, the recognition and consideration of which are important when emerging research are taken into account in clinical decisions. Further, it is worth noting that the data included in meta-analysis are largely retrospective in nature, and this is highlighted by the authors, who emphasise that subgroup analysis was prevented due to the absence of raw patient data - an unavoidable obstacle in this instance. ${ }^{1}$ It should also be emphasised that performing large-scale, randomised prospective trials are especially challenging (and possibly unethical) in the field of cardiac surgery and specifically aortic repair. ${ }^{5}$

Optimal medical therapy (OMT) for un-TBAD, as emphasised by Howardet al. and described by Kaji, is usually the first-line treatment for TBAD, and aims to control blood pressure to attenuate haemodynamic shear forces exerted on the aortic intima, to prevent aortic structural compromise. ${ }^{1,3}$ To this end, various antihypertensives, negative inotropic agents, and vasodilators are prescribed in conjunction with lifestyle changes. ${ }^{1,3,4}$ Whereas patients with acute co-TBAD are also treated with TEVAR, the use thereof in unTBAD is a matter of debate - sudden death, severe disease progression, and the need for late intervention are documented omnipresent risks in un-TBAD patients managed with OMT alone. ${ }^{2,8,9}$ Additionally, landmark studies including INSTEAD, ADSORB, and VIRTUE all suggest that aortic remodelling and false lumen thrombosis is augmented by TEVAR in patients with both un-TBAD and co-TBAD. ${ }^{9}, 10,{ }^{11}$ Critically though, the INSTEAD trial suggested that TEVAR did not lead to improved 2-year survival in un-TBAD patients. ${ }^{9}$ The authors question whether treating un-TBAD with TEVAR would be a step forward in the management of high-risk un-TBAD patients - un-TBAD is a complex syndrome to manage, and a multidisciplinary approach is warranted. ${ }^{1,3}$ TEVAR for acute-phase co-TBAD, on the other hand, is broadly recognised as effective and has overtaken open surgical intervention as the mainstay of aortic repair for TBAD. ${ }^{12}$ Zeeshan et al. highlight that TEVAR for co-TBAD is associated with lower rates of in-hospital and 30-day mortality than both open surgical repair and OMT, as well as improved rates of survival 1-, 3-, and 5-years postintervention. ${ }^{12}$ Similar findings have been echoed by other large-scale investigations, including ADSORB and VIRTUE. ${ }^{10,11}$ Indeed, further prospective, randomised multi-centre studies would be of immense benefit towards helping clinicians decide whether at-risk un-TBAD patients should be managed in a similar fashion to their co-TBAD counterparts.

To conclude, the findings in Howard and colleagues' manuscript suggest that despite notably higher rates of co-morbidity in patients with co-TBAD than un-TBAD, there was no statistically significant difference in in-hospital mortality or 5-year survival, however 30-day mortality appeared to be higher in the co-TBAD cohort. ${ }^{1}$ Patients with co-TBAD appeared to be at an increased risk of postoperative complications such as stroke or TEVAR endoleak, while un-TBAD patients were at a comparatively greater risk of renal failure. ${ }^{1}$ The manuscript presents a robust systematic review that benefits from sound statistical methods and analyses, and therefore provides valuable and useful insight into the comparative efficacies of TEVAR for un-TBAD and co-TBAD. 


\section{References}

Howard C, Sheridan J, Bashir M, Idhrees M, Iddawela S, George M, Dias D, Srinivasan A, Picca L, Smith T, Reza S, Calow R, Munir W, Cross O. TEVAR for Complicated \& Uncomplicated Type B Aortic Dissection - Systematic Review and Meta-Analysis. Journal of Cardiac Surgery. 2021.

Zhu T, Si Y, Fang Y, Chen B, Yang J, Jiang J, Yue J, Fu W. Early outcomes of the conformable stent graft for acute complicated and uncomplicated type B aortic dissection. Journal of vascular surgery. 2017 Dec $1 ; 66(6): 1644-52$.

Kaji S. Update on the therapeutic strategy of Type B aortic dissection. Journal of atherosclerosis and thrombosis. 2017:RV17017.

Nauta FJ, Trimarchi S, Kamman AV, Moll FL, Van Herwaarden JA, Patel HJ, Figueroa CA, Eagle KA, Froehlich JB. Update in the management of type B aortic dissection. Vascular Medicine. 2016 Jun;21(3):25163.

Lin L, Chu H. Quantifying publication bias in meta-analysis. Biometrics. 2018 Sep;74(3):785-94.

Lin L, Chu H, Murad MH, Hong C, Qu Z, Cole SR, Chen Y. Empirical comparison of publication bias tests in meta-analysis. Journal of general internal medicine. 2018 Aug;33(8):1260-7.

Mousavizadeh M, Daliri M, Aljadayel HA, Idhrees M, Rezaei Y, Bashir M, Hosseini S. Hypothermic Circulatory Arrest Time affects neurological outcomes of Frozen Elephant Trunk for Acute Type A Aortic Dissection: a systematic review and meta-analysis.

Tsai TT, Trimarchi S, Nienaber CA. Acute aortic dissection: perspectives from the International Registry of Acute Aortic Dissection (IRAD). European journal of vascular and endovascular surgery. 2009 Feb 1;37(2):149-59.

Nienaber CA, Zannetti S, Barbieri B, Kische S, Schareck W, Rehders TC. INvestigation of STEnt grafts in patients with type B Aortic Dissection: design of the INSTEAD trial - a prospective, multicenter, European randomized trial. American heart journal. 2005 Apr 1;149(4):592-9.

Brunkwall J, Kasprzak P, Verhoeven E, Heijmen R, Taylor P, Alric P, Canaud L, Janotta M, Raithel D, Malina M, Resch T. Endovascular repair of acute uncomplicated aortic type B dissection promotes aortic remodelling: 1 year results of the ADSORB trial. European Journal of Vascular and Endovascular Surgery. 2014 Sep 1;48(3):285-91.

VIRTUE Registry Investigators. Mid-term outcomes and aortic remodelling after thoracic endovascular repair for acute, subacute, and chronic aortic dissection: the VIRTUE Registry. European Journal of Vascular and Endovascular Surgery. 2014 Oct 1;48(4):363-71.

Zeeshan A, Woo EY, Bavaria JE, Fairman RM, Desai ND, Pochettino A, Szeto WY. Thoracic endovascular aortic repair for acute complicated type B aortic dissection: superiority relative to conventional open surgical and medical therapy. 